\title{
Hollywoodfilmtitel im Deutschen und im Thailändischen
}

\author{
Pasinee Seenlabut und Korakoch Attaviriyanupap (Nakhon Pathom)
}

\begin{abstract}
This paper is a contrastive analysis which explores how the English Hollywood film titles are translated into German and Thai. The purpose of the study is to answer the following questions: 1) What is the difference between the length of the film titles in the original English version and in their German and Thai translation; 2) Which translation methods can be found in the translation of the Hollywood film titles into German and Thai?; 3) Which constructions can be found in the translated film titles in German and in Thai in comparison to the original ones? The study is based on a corpus of 900 film titles including 300 of Hollywood film titles and their translation in German and Thai. It is found that the length of the film titles is between one and twelve words. While film titles in both European languages contain mostly two words, the use of four-word titles occurs most frequently in the Thai translation. 10 translation strategies are found in total. In German, the original English titles are mostly kept. In contrast to German, the titles are mostly totally changed in Thai. Regarding the structure of the film titles, there are totally 7 categories. Nominal phrase is the most frequently found construction of film titles in all these three analyzed languages.
\end{abstract}

\section{$1 \quad$ Einleitung}

Warum wurde der englische Hollywood-Filmtitel The Revenant ins Thailändische als tว̌nrǒ:t (,müssen“ ,überleben'): Überleben ist ein Muss übertragen? Wieso erscheint dieser Titel bei der Übersetzung ins Deutsche als Der Rückkehrer? Aus welchem Grund ist die Länge der ursprünglich englischen Titel im Vergleich zu denjenigen im Thailändischen und im Deutschen häufig unterschiedlich? Welche Strukturen bzw. Konstruktionen weisen Filmtitel in den drei Sprachen auf?

Die obenstehenden Fragen betreffen die Filmtitelübersetzung, die in der Filmindustrie seit langem eine wichtige Rolle spielt, da zahlreiche englischsprachige Hollywoodfilme weltweit exportiert werden und dadurch in verschiedene Zielsprachen übersetzt werden müssen. Filmtitel haben üblicherweise eine Appellfunktion, weil sie das erste sind, was das Zielpublikum dazu bewegt, die Inhalte der Originalfilme wahrzunehmen (cf. Thongwan 2012: 4). Die Übersetzung ermöglicht also eine kommunikative Handlung, die sonst aufgrund von Sprach- und Kulturbarrieren verhindert werden könnte (cf. Nord 1991: 31). Neben der Übersetzung von Filmtiteln bilden die syntaktischen Strukturen der Titel einen weiteren interessanten Untersuchungsaspekt, weil sie bestimmte Eigenschaften der betroffenen Sprachen aufweisen. Zusätzlich dazu könnte die Wörteranzahl der übersetzten Filmtitel in verschiedenen Sprachen relevant sein. Die

Linguistik online 101, 1/20 - http://dx.doi.org/10.13092/lo.101.6674

CC by 3.0 
Übersetzungsstrategien, die syntaktische Struktur der Titel und die Anzahl der Wörter im Filmtitel sind deshalb drei erforschenswerte Aspekte.

Obwohl es bereits Untersuchungen der Filmtitel gibt (cf. etwa Schubert 2004; Bouchehri 2008; Brückner 2012; Thongwan 2012 und Masae et al. 2018), lassen sich ausschließlich Studien von Filmtitelübersetzungen der englischen Sprache nur entweder in eine andere Sprache (Deutsch oder Thailändisch) oder mit zwei anderen europäischen Sprachen (Deutsch und Französisch) finden. Es liegt keine Untersuchung vor, in der gleichzeitig zwei Zielsprachen, die verschiedenen Sprachfamilien entstammen, kontrastiv analysiert werden.

In der vorliegenden Untersuchung sollen die folgenden drei Fragen beantwortet werden:

1. Wie unterscheidet sich die Länge der Filmtitel in der Übersetzung von den Originalfilmtiteln?

2. Welche Methoden lassen sich beim Übersetzen der Hollywoodfilmtitel ins Deutsche und ins Thailändische finden?

3. Welche Konstruktionstypen kommen in den übersetzten Filmtiteln im Deutschen und im Thailändischen vor?

Das nächste Kapitel beschäftigt sich mit dem allgemeinen theoretischen Rahmen und Forschungsstand. Danach folgt die Darstellung des analysierten Korpus und des methodischen Vorgehens der vorliegenden Studie. Die Ergebnisse werden in Anlehnung an den oben gestellten Fragen Schritt für Schritt präsentiert und diskutiert. Das Fazit und der Ausblick folgen danach im Schlusskapitel.

\section{Theoretischer Rahmen und Forschungsstand}

\subsection{Methoden der Übersetzung von Filmtiteln}

Bei der Recherche relevanter Literatur sind Übersetzungsmethoden bzw. Translationsverfahren von Filmtiteln in insgesamt 10 verschiedenen Formen bzw. 10 Modellen festgestellt worden, die sich in der nachstehenden Tabelle darstellen lassen:

\begin{tabular}{|l|c|c|c|c|c|}
\hline Übersetzungsmethode $^{1}$ & $\begin{array}{c}\text { Schubert } \\
\text { (2004) }\end{array}$ & $\begin{array}{c}\text { Bouchehri } \\
\text { (2008) }\end{array}$ & $\begin{array}{c}\text { Brückner } \\
\text { (2012) }\end{array}$ & $\begin{array}{c}\text { Thongwan } \\
\text { (2012) }\end{array}$ & $\begin{array}{c}\text { Masae } \\
\text { et al. } \\
\text { (2018) }\end{array}$ \\
\hline $\begin{array}{l}\text { 1. Wort-für-Wort- } \\
\text { Übersetzung }\end{array}$ & $\checkmark$ & $\checkmark$ & $\checkmark$ & $\checkmark$ & $\checkmark$ \\
\hline 2. Reduktion & $\checkmark$ & $\checkmark$ & $\checkmark$ & & \\
\hline 3. Addition & $\checkmark$ & $\checkmark$ & $\checkmark$ & $\checkmark$ & $\checkmark$ \\
\hline 4. Substitution & $\checkmark$ & & & & \\
\hline $\begin{array}{l}\text { 5. Umtitelung in der } \\
\text { Zielsprache }\end{array}$ & $\checkmark$ & $\checkmark$ & $\checkmark$ & $\checkmark$ & $\checkmark$ \\
\hline
\end{tabular}

\footnotetext{
${ }^{1}$ Die Benennung jeder einzelnen Übersetzungsmethode von Filmtiteln kann Unterschiede aufweisen, obwohl es sich um dasselbe Verfahren handelt. Beispielsweise benutzte Bouchehri (2008) „Titelinnovation“, während Schubert (2004) „Umtitelung“ verwendete. Für die vorliegende Arbeit wird die Bezeichnung bevorzugt, die einfacher $\mathrm{zu}$ verstehen ist.
} 


\begin{tabular}{|l|c|c|c|c|c|}
\hline Übersetzungsmethode $^{1}$ & $\begin{array}{c}\text { Schubert } \\
\text { (2004) }\end{array}$ & $\begin{array}{c}\text { Bouchehri } \\
\text { (2008) }\end{array}$ & $\begin{array}{c}\text { Brückner } \\
\text { (2012) }\end{array}$ & $\begin{array}{c}\text { Thongwan } \\
\text { (2012) }\end{array}$ & $\begin{array}{c}\text { Masae } \\
\text { et al. } \\
\text { (2018) }\end{array}$ \\
\hline $\begin{array}{l}\text { 6. Umtitelung in der } \\
\text { Originalsprache }\end{array}$ & $\checkmark$ & & & & \\
\hline 7. Titelidentität & $\checkmark$ & $\checkmark$ & $\checkmark$ & $\checkmark$ & $\checkmark$ \\
\hline 8. Teiltitelidentität & & $\checkmark$ & & $\checkmark$ & $\checkmark$ \\
\hline $\begin{array}{l}\text { 9. Hybridform mit } \\
\text { Einfachtitel }\end{array}$ & & $\checkmark$ & $\checkmark$ & $\checkmark$ & $\checkmark$ \\
\hline $\begin{array}{l}\text { 10. Hybridform mit } \\
\text { Titelgefüge }\end{array}$ & & & & \\
\hline
\end{tabular}

Tabelle 1: Übersetzungsmethoden in der gängigen Literatur

Bei der Wort-für-Wort-Übersetzung bleiben die sprachliche Struktur und die Bedeutung der übersetzten Filmtitel unverändert. Es bezieht sich auf jene identischen oder analogen Titel, bei denen nur minimale Eingriffe vorgenommen wurden, wie z. B. Veränderungen der Interpunktion, der Akzentsetzung, des Tempus, der Reihenfolge von Wörtern oder die Umwandlung von der Singularform eines Wortes in die zugehörige Pluralform (cf. Bouchehri 2008: 73).

Reduktion bzw. Subtraktion bedeutet die Verkürzung und Vereinfachung eines Originaltitels. Nach Schubert (2004: 242) kann die Subtraktion die Funktion haben, potentiell verwirrende Details auszusparen.

Bei der Addition werden dem übersetzten Titel explizit Informationen hinzugefügt, ohne dass eine Transformation stattfindet (cf. Schubert 2004: 245). Zudem kann die Addition als translatorisches Hilfsmittel zur Auflösung von Polysemien und anderen Sprachspielen genutzt werden (cf. Bouchehri 2008: 77). Dahingegen wird bei der Substitution der Filmtitel nur ein Teil des Originals wörtlich übersetzt, während der andere Teil durch einen frei gewählten Ausdruck in der Zielsprache ersetzt wird (cf. Schubert 2004: 243).

Die Umtitelung bezieht sich auf die Übersetzungsform, bei der dem Film ein neuer Titel gegeben wird, der sich vollständig von der Überschrift in der Originalsprache unterscheidet. Es entsteht ein neuer Titel für die übersetzte Version des Kinofilmes, wobei hier keinerlei Äquivalenzbeziehung zwischen Ausgangs- und Zieltext bestehen muss (cf. Bouchehri 2008: 78). Zwei Subkategorien liegen vor: Umtitelung in der Zielsprache und Umtitelung in der Originalsprache. Prinzipiell soll das Verständnis des Zielpublikums gefördert werden. Bei der Umtitelung in der Originalsprache handelt es sich meistens um englischen Grundwortschatz (cf. Schubert 2004: 249).

Unter Titelidentität versteht man die Beibehaltung der Originaltitel. Titelidentitäten liegen nahe bei großem Bekanntheitsgrad des Filmes, aufgrund der Kürze solcher Titel oder schlicht wegen ihrer Unübersetzbarkeit (cf. ibd.: 252). Wenn aber nur ein Teil der Originalfilmtitel transkribiert wird und es keine Erweiterung in dem neuen übersetzten Titel gibt, liegt eine Teiltitelidentität vor (cf. Thongwan 2012: 41).

Werden Filmtitel mit mehreren Übersetzungsmethoden übertragen, handelt es sich um die sogenannte Titelhybridform. Hier liegen zwei Subkategorien vor. Während bei der Hybridform 
mit Einfachtitel nur ein Teil des Titels analog übersetzt wird und der andere Teil im Original beibehalten wird (Bouchehri 2008: 83), wird bei der Hybridform mit Titelgefüge der ganze Originalfilmtitel übersetzt und mit einem Zusatz in der Zielsprache ergänzt (cf. Masae et al. 2018: 115).

\subsection{Forschungsstand}

Bei der Betrachtung der Filmtitelübersetzungsmethoden liegen mehrere Vorläufer der hier durchgeführten Studie vor. Fünf ausgewählte relevante wissenschaftliche Studien werden im Folgenden zusammengestellt.

In der Studie von Schubert (2004) wurden 1.486 englische Filmtitel und ihre deutschsprachigen Neutitel, die im Jahr 1944 bis 2002 produziert wurden, untersucht. Es wurde festgestellt, dass von 1940 bis 1950 die wörtliche Übersetzung am häufigsten verwendet wurde. Hierauf folgte in den Jahren 1970 bis 2002 die Methode der Titelidentität.

Bouchehri (2008) verglich mehr als 1.500 englischsprachige Originalfilmtitel mit deutschen und französischen Übersetzungsfilmtiteln, die von 1920 bis 2000 erschienen. Die Methode Titelinnovation wird in deutschen Übersetzungstiteln am häufigsten benutzt, während in der Übersetzung ins Französische größtenteils Titelidentitäten angewendet wurden. Hybridformen werden am wenigsten in beiden Sprachen verwendet. Außerdem untersuchte Bouchehri noch zwei weitere Aspekte: die Titellänge und die syntaktische Titelstruktur. Ein-Wort-Titel finden sich am häufigsten bei Titelidentitäten und Drei-Wort-Titel am häufigsten bei Titelinnovationen. Bei Titelanalogien werden Fünf-Wort-Titel am häufigsten gezeigt. Im Hinblick auf die syntaktische Titelstruktur lassen sich sechs Kategorien finden (cf. Nord 1993: 59, zitiert nach Bouchehri 2008: 46), die auf folgende Formen zurückgehen: nominale Titel, satzförmige Titel, adverbiale Titel, adjektivische Titel, verbale Titel und interjektionsförmige Titel. Der nominale Titel wird in den Filmtiteln aller drei untersuchten Sprachen am häufigsten angewendet.

Brücker (2012) untersuchte die Originalfilmtitel im Französischen und die Übersetzung ins Deutsche. Sie benutzte 3.200 französischsprachige Filmtitel, die im Jahr 1933 bis 2011 produziert wurden. Die Übersetzungsstrategien von Bouchehri (2008) verwendete die Forscherin als ihr Modell. Bei ihren Daten wurde die Titelinnovation am meisten benutzt, während die Titelidentität am wenigsten angewendet wurde.

Thongwan (2012) analysierte 98 ins Thailändische übersetzte amerikanische Komödienfilmtitel, die von 2008 bis 2010 produziert wurden. Zehn Übersetzungsmethoden wurden gefunden. Daraus ergibt sich, dass Titelinnovationen mit neuen Wörtern am beliebtesten waren, während Teiltitelidentitäten und Titelanalogien am wenigsten benutzt wurden.

Masae et al. (2018) verglichen und analysierten 102 Hollywoodfilmtitel, die ins Thailändische übersetzt wurden. Es handelte sich um Filme aus dem Jahr 2016 und 2017. Sie variierten den methodischen Ansatz von Thongwan (2012) und fanden eine neue Methode. Insgesamt wurden 11 Übersetzungsmethoden gefunden. Titelidentitäten treten dabei am häufigsten auf und Titelanalogien und Teiltitelidentitäten mit Erweiterung kommen am seltensten vor. 


\section{Das analysierte Korpus und das methodische Vorgehen}

In dieser Arbeit wurden die analysierten Filmtitel aus der Hollywood-Website IMDB.com übernommen und eigens als Korpus zusammengestellt. Das Korpus erhält insgesamt 900 Titel von 300 Hollywoodfilmen, die 2015 bis 2017 produziert wurden. Es besteht aus jeweils 300 Originalfimtiteln, deren deutschen Übersetzungstiteln und deren thailändischen Versionen. Die gesammelten Filmtitel werden zwecks der Analyse aufbereitet, um sie im Hinblick auf die Titellänge, die Übersetzungsmethode, und sprachliche Konstruktionstypen kategorisieren zu können. Die Codierung besteht aus der Abkürzung von jener Sprache, d. h. Deutsch = D, Thailändisch $=\mathrm{T}$ und Englisch bzw. Originaltitel = E, gefolgt von dem Jahr (2015, 2016 und 2017) und der Nummer des Filmtitels im betroffenen Jahr. Bei der Titellänge bzw. der Zählung der Wörter im Titel wird die Zählfunktion des Word-Programms verwendet, das in allen drei untersuchten Sprachen vergleichsweise ähnlich funktioniert. Die Kategorisierung der Übersetzungsmethode bezieht sich ausschließlich auf Tabelle 1. Die Struktur der Titel wird im Hinblick auf die sprachlichen Konstruktionstypen in 7 Kategorien unterschieden: Nominalphrase (NP), Verbalphrase (VP), Adjektivphrase (AdjP), Adverbphrase (AdvP), Präpositionalphrase (PP), Satz (S) und Interjektion (Interj).

Alle analysierten Filmtitel werden mit der oben beschriebenen Codierung annotiert, wie beispielsweise in der folgenden Tabelle dargestellt wird.

\begin{tabular}{|c|c|c|c|}
\hline Titel-Nr. & Titellänge & $\begin{array}{c}\text { Übersetzungsme- } \\
\text { thode }\end{array}$ & $\begin{array}{c}\text { Konstrukions- } \\
\text { typen }\end{array}$ \\
\hline D_2015_1 & 4 & 6 & AdjP \\
\hline T_2015_1 & 5 & 5 & AdjP \\
\hline E_2015_1 & 2 & - & AdjP \\
\hline
\end{tabular}

Tabelle 2: Die verwendeten Codes für die Analyse

\section{$4 \quad$ Ergebnisse der Korpusanalyse}

\subsection{Länge von Filmtiteln}

Im untersuchten Korpus enthalten die Filmtitel in den drei Sprachen eins bis zwölf Wörter, wie in der folgenden Tabelle dargestellt wird:

\begin{tabular}{|c|c|c|c|c|c|c|}
\hline \begin{tabular}{c} 
Länge $\begin{array}{c}\text { der } \\
\text { Filmtitel } \\
\text { (Anzahl } \\
\text { der } \\
\text { Wörter) }\end{array}$ \\
\cline { 2 - 7 }
\end{tabular} & \multicolumn{2}{|c|}{ Originalfilmtitel } & \multicolumn{2}{|c|}{ Deutsche Version } & \multicolumn{2}{c|}{ Thai-Version } \\
\hline 1 & 69 & 23 & 53 & 17,67 & 8 & 2,67 \\
\hline 2 & 99 & 33 & 86 & 28,67 & 14 & 4,67 \\
\hline 3 & 59 & 19,67 & 62 & 20,67 & 66 & 22 \\
\hline 4 & 31 & 10,67 & 49 & 16,33 & 76 & 25,33 \\
\hline 5 & 23 & 7,67 & 23 & 7,67 & 47 & 15,67 \\
\hline
\end{tabular}




\begin{tabular}{|c|c|c|c|c|c|c|}
\hline $\begin{array}{c}\text { Länge } \\
\text { der } \\
\text { Filmtitel } \\
\text { (Anzahl } \\
\text { der } \\
\text { Wörter) }\end{array}$ & \multicolumn{2}{|c|}{ Originalfilmtitel } & \multicolumn{2}{|c|}{ Deutsche Version } & \multicolumn{2}{c|}{ Thai-Version } \\
\cline { 2 - 7 } & Anzahl & $\begin{array}{c}\text { Prozentualer } \\
\text { Anteil }\end{array}$ & Anzahl & $\begin{array}{c}\text { Prozentualer } \\
\text { Anteil }\end{array}$ & Anzahl & $\begin{array}{c}\text { Prozentualer } \\
\text { Anteil }\end{array}$ \\
\hline 6 & 9 & 3 & 17 & 5,67 & 51 & 17 \\
\hline 7 & 6 & 2 & 7 & 2,33 & 17 & 5,67 \\
\hline 8 & 2 & 0,67 & 2 & 0,67 & 14 & 4,67 \\
\hline 9 & 1 & 0,33 & - & 0 & 3 & 1 \\
\hline 10 & - & 0 & - & 0 & 3 & 1 \\
\hline 12 & - & 0 & 1 & 0,33 & 1 & 0,33 \\
\hline & 300 & 100 & 300 & 100 & 300 & 100 \\
\hline
\end{tabular}

Tabelle 3: Vergleich der Filmtitellänge in den drei untersuchten Sprachen

In Bezug auf die Titellänge lässt sich anhand der Ergebnisse in der obigen Tabelle belegen, dass die englischen Originalfilmtitel am häufigsten zwei Wörter enthalten (33\%). Zwei-WortTitel werden im Deutschen ebenfalls am häufigsten gefunden $(28,7 \%)$. Im Gegensatz dazu wird ein Vier-Wort-Titel im Thailändischen am häufigsten verwendet $(25,3 \%)$, und Filmtitel mit zwei Wörtern lassen sich viel seltener finden (4,67\%). Bemerkenswert ist außerdem, dass der längste englische Originaltitel nur neun Wörter enthält, während sich Zwölf-Wort-Titel sowohl im Deutschen als auch im Thailändischen finden lassen, obwohl deren Anzahl nur minimal ist. Längere Filmtitel scheinen im Thailändischen üblicher zu sein.

Wie sich nach der obigen Tabelle zeigen lässt, werden bei englischen Originaltiteln und bei den übersetzten Filmtiteln im Deutschen häufig nur ein Wort oder zwei Wörter verwendet. Ein Zwei-Wort-Titel wie beispielsweise Finding Dory (E_2016_21) wird ins Deutsche auch mit zwei Wörtern als Findet Dorie (D_2016_21) übersetzt. Im Gegensatz dazu werden bei übersetzten Filmtiteln im Thailändischen meistens mehr Wörter benutzt, deswegen sind die thailändischen Filmtitel länger als die Originalfilmtitel, zum Beispiel Don't Breathe (E_2016_92) erscheint im Thailändischen als lomhă:jcaj săy ta:j (,Atem‘ ,befehlen“ ,sterben“): ,Atemzug mit Todesbefehl'.

In der vorliegenden Untersuchung wird nur ein englischer Filmtitel mit neun Wörtern aufgezeigt: Pirates of The Caribbean: Dead Men Tells No Tales (E_2017_56). Daneben werden in der deutschen und thailändischen Übersetzung Zwölf-Wort-Titel als die längsten gefunden. In beiden Sprachen liegt jedoch nur jeweils ein Beispiel vor:

Wenn du stirbst, zieht dein ganzes Leben an dir vorbei, sagen sie (D_2017_45)

$\begin{array}{lllll}\text { phajrè:t } & \grave{j}: f & d \grave{r} ? & k h \varepsilon: r i ́ p b \hat{\imath}: a n & \text { phâ:k hâ: } \\ \text { pirate } & \text { of } & \text { the } & \text { Caribbean } & \text { Episode } 5 \\ \text { sǒykhra:m } & k h \varepsilon ́: n & \text { co:nsalà:t } & \text { ráj } & \text { chî:p }\left(\mathrm{T} \_2017 \_56\right) \\ \text { Krieg } & \text { Rache } & \text { Pirat } & \text { ohne } & \text { Leben } \\ \text { 'Pirates of the Caribbean Teil 5: Rachenkrieg des leblosen Piraten' }\end{array}$




\subsection{Verwendete Übersetzungsmethoden}

In der vorliegenden Untersuchung werden alle Hollywoodfilmtitel nach den in Kapitel 2 dargestellten Übersetzungsverfahren analysiert. Bei den untersuchten deutschen und thailändischen Filmtiteln lassen sich 10 Übersetzungsmethoden finden, die in der folgenden Tabelle dargelegt sind:

\begin{tabular}{|c|c|c|c|c|}
\hline \multirow{2}{*}{ Übersetzungsmethode } & \multicolumn{2}{|c|}{ Deutsche Version } & \multicolumn{2}{|c|}{ Thai-Version } \\
\hline & Anzahl & $\begin{array}{c}\text { Prozentualer } \\
\text { Anteil }\end{array}$ & Anzahl & $\begin{array}{c}\text { Prozentualer } \\
\text { Anteil }\end{array}$ \\
\hline $\begin{array}{l}\text { 1. Wort-für-Wort- } \\
\text { Übersetzung }\end{array}$ & 29 & 9,67 & 14 & 4,67 \\
\hline 2. Reduktion & 6 & 2 & 4 & 1,33 \\
\hline 3. Addition & 1 & 0,33 & 39 & 13 \\
\hline 4. Substitution & 3 & 1 & 9 & 3 \\
\hline $\begin{array}{l}\text { 5. Umtitelung in der } \\
\text { Zielsprache }\end{array}$ & 33 & 11 & 123 & 41 \\
\hline $\begin{array}{l}\text { 6. Umtitelung in der } \\
\text { Originalsprache }\end{array}$ & 6 & 2 & 5 & 1,67 \\
\hline 7. Titelidentität & 175 & 58,33 & 48 & 16 \\
\hline 8. Teiltitelidentität & 6 & 2 & 1 & 0,33 \\
\hline $\begin{array}{l}\text { 9. Hybridform mit } \\
\text { Einfachtitel }\end{array}$ & 9 & 3 & 19 & 6,33 \\
\hline $\begin{array}{l}\text { 10. Hybridform mit Ti- } \\
\text { telgefüge }\end{array}$ & 26 & 8,67 & 25 & 8,33 \\
\hline Sonstiges & 6 & 2 & 13 & 4,33 \\
\hline & 300 & 100 & 300 & 100 \\
\hline
\end{tabular}

Tabelle 4: Gefundene Übersetzungsmethoden in den zwei übersetzten Sprachen

Die obenstehende Tabelle zeigt im Hinblick auf die verwendeten Übersetzungsmethoden, dass die Titelidentität in der deutschsprachigen Sprache am häufigsten verwendet ist $(58,33 \%)$, gefolgt von Umtitelung in der Zielsprache mit $11 \%$ und Wort-für-Wort-Übersetzung mit 9,67\% . Die am seltensten gefundene Translationsform ist Addition, die nur bei einem Filmtitel $(0,33 \%)$ auftritt.

An der ersten Stelle findet man im Deutschen die Beibehaltung der Originalfilmtitel, wie im Fall von 13 Hours: The Secret soldiers of Benghazi (E_2016_1; D_2016_1). Als nächstes beleuchtet die zweite Translationsform die Umtitelung in der Zielsprache, wie im Fall von The Intern (E_2015_40) vs. Man lernt nie aus (D_2015_40). Auf Platz drei liegt ein Titel vor, der sich der Wort-für-Wort-Übersetzung zuordnen lässt, z. B. The Martian (E_2015_85) vs. Der Marsianer (D_2015_85). Die Addition wird als letztes Übersetzungsverfahren gefunden, wie bei Anthropoid (E_2016_65) der Fall ist. Die deutsche Fassung heißt Operation Anthropoid (D_2016_65).

Im Kontrast dazu liegt bei thailändischen Filmtiteln die Umtitelung in der Zielsprache mit 41 Prozent aller analysierten Hollywood-Filmtitel an der Spitze. Die Beibehaltung der 
Originalsprache gilt mit 48 Filmtiteln (16\%) als die zweitbeliebteste Methode. Auf Platz drei findet man die Addition mit 13\%. Dem gegenüber wurde die Teiltitelidentität in thailändischen übersetzten Titeln am wenigsten verwendet $(0,33 \%)$. Als erstes wird die Umtitelung in der Zielsprache bzw. ins Thailändische dargestellt:

The Girl on the Train (E_2016_101)

pom lò:n ra:y ms:rana? (T_2016_101)

Knoten spukhaft Schiene tot

'Der spukhafte Knotenpunkt: Die tote Schiene'

Des Weiteren liegen Beispiele von identischen Filmtiteln vor. Dies zeigt etwa der Titel Bridget Jones's Baby (T_2017_76). Auf dem dritten Platz sind Filmtitel mit Addition, wie zum Beispiel:

The Shallow (E_2016_102)

narók nám tû:n (T_2016_102)

Hölle Wasser untief

'Der Teufel der Untiefe'

Schließlich wird die Teiltitelidentität im Thailändischen kaum verwendet. Im folgenden Beispiel sieht man, dass nicht der ganze Titel übernommen wird:

Batman v Superman: Dawn of Justice (E_2016_83)

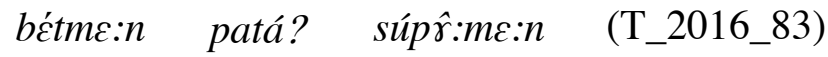

Batman gegen Superman

'Batman gegen Superman'

Bei den Formen der Übersetzung werden zehn gleiche Übersetzungsmethoden in beiden analysierten Sprachen gefunden, aber die prozentualen Anteile der untersuchten Filmtitel sind unterschiedlich. Wie in der obigen Beschreibung liegt die Beibehaltung der englischen Filmtitel auf dem ersten Platz im Deutschen. Im Gegensatz dazu wird in der thailändischen Sprache die Umtitelung in der Zielsprache an erster Stelle aufgezeigt. Diese Methode gilt hingegen als die zweite überwiegende Translationsstrategie im Deutschen, während Titelidentitäten im Thailändischen an zweiter Stelle stehen. Auch bei der dritten Stelle liegt eine Abweichung in den beiden Zielsprachen vor: die Wort-für-Wort-Übersetzung im Deutschen vs. die Addition im Thailändischen. Hinzu kommt, dass die am wenigsten verwendete Methode bei der deutschen Sprache die Addition ist. Hingegen wird im Thailändischen Teiltitelidentität am seltensten festgestellt.

Wie sich ebenfalls aus Tabelle 4 ablesen lässt, gibt es noch ein Translationsverfahren in den zwei Sprachen, das in der vorliegenden Studie als „Sonstiges“ kategorisiert wird. Es handelt sich hier um die Mischung verschiedener Übersetzungsmethoden. Diese Formen werden in der vorliegenden Studie als fünf weitere Übersetzungsstrategien festgestellt, weil sie nicht unter die obenstehenden Hybridformen fallen.

Die erste gemischte Form ist Wort-für-Wort-Übersetzung mit Titelgefüge. Gemeint ist das Titelgefüge hierbei als eine Ergänzung einer Filmüberschrift, die Wort für Wort in die deutsche Sprache übersetzt ist, mit einem Zusatz am Titelende, beispielsweise markiert durch einen Gedankenstich oder einen Doppelpunkt, wie im Falle von Gifted (E_2017_48) vs. Begabt - Die Gleichung eines Lebens (D_2017_48) zu sehen ist. 
Zudem tritt die Substitution in der Originalsprache mit Titelgefüge auf. Der Filmtitel wird nur bei einem englischen Wort verändert und im Anschluss daran wird eine deutsche Phrase hinzugefügt: Fifty Shades Darker (E_2017_76) vs. Fifty Shades of Grey - Gefährliche Liebe (D_2017_76).

Bei der Reduktion mit Titelgefüge handelt es sich um die Mischung, bei der nur ein Teil des Originalfilmtitels übersetzt und ein englisches Wort dem deutschen übersetzten Titel als Titelgefüge ergänzt wird: War of The Planet of The Apes (E_2017_85) vs. Planet der Affen: Survival (D_2017_85). Diese Methode lässt sich ebenfalls im Thailändischen finden:

Valerian and The City of A Thousand Planets (E_2017_58)

wa:le:rî:an phlík càkawa:n (T_2017_58)

Valerian umdrehen Universum

'Valerian: Universum umdrehen'

Wenn ein Teil des Originaltitels beibehalten wird, während ein zusätzlicher Untertitel dazugegeben wird, lässt sich der Übersetzungstitel der Teiltitelidentität mit Titelgefüge zuordnen:

Atomic Blonde (E_2017_9)

$\begin{array}{llll}\text { blon } & \text { sǔ:aj } & \text { kracui } & \text { (T_2017_9) } \\ \text { blond } & \text { schön } & \text { zerstreut } & \end{array}$

'Die Blonde verstreut ihre Schönheit'

Anders als bei den anderen oben genannten Mischformen mit Titelgefüge handelt es sich bei der letzten Kategorie um die Reduktion mit Zusatz, weil die hinzugefügte Information nicht am Ende, sondern in den Übersetzungstitel integriert wird, wie im folgenden Fall zu sehen ist:

Ouija: Origin of Evil (E_2016_69)

kamr̀t krada:n pi:sà:t (T_2016_69)

geboren Brett Teufel

'Ursprung des Teufelsbretts'

\subsection{Konstruktionstypen von Filmtiteln}

Eine syntaktische Untersuchung des Korpus liefert folgende Ergebnisse, die zunächst anhand einer tabellerischen Darstellung gezeigt werden:

\begin{tabular}{|c|c|c|c|}
\hline \multirow{2}{*}{ Konstruktionstyp } & \multicolumn{3}{|c|}{$\begin{array}{c}\text { Anzahl und prozentualer Anteil der ver- } \\
\text { schiedenen Konstruktionstypen }\end{array}$} \\
\cline { 2 - 4 } & Deutsch & Thailändisch & Englisch \\
\hline NP & $194(64,67 \%)$ & $119(39,67 \%)$ & $\begin{array}{c}223 \\
(74,33 \%)\end{array}$ \\
\hline VP & $12(4 \%)$ & $29(9,67 \%)$ & $12(4 \%)$ \\
\hline AdjP & $9(3 \%)$ & $7(2,33 \%)$ & $6(2 \%)$ \\
\hline PP & $3(1 \%)$ & $4(1,33 \%)$ & $2(0.67 \%)$ \\
\hline
\end{tabular}




\begin{tabular}{|c|c|c|c|}
\hline \multirow{2}{*}{ Konstruktionstyp } & \multicolumn{3}{|c|}{$\begin{array}{c}\text { Anzahl und prozentualer Anteil der ver- } \\
\text { schiedenen Konstruktionstypen }\end{array}$} \\
\cline { 2 - 4 } & Deutsch & Thailändisch & Englisch \\
\hline S & $13(4,33 \%)$ & $48(16 \%)$ & $19(6,33 \%)$ \\
\hline M & $67(22,33 \%)$ & $93(31 \%)$ & $35(11,67 \%)$ \\
\hline & $300(100 \%)$ & $300(100 \%)$ & $300(100 \%)$ \\
\hline
\end{tabular}

Tabelle 5: Konstruktionstypen der Filmtitel in den drei Sprachen

Anhand der obenstehenden Tabelle ist ersichtlich, dass die Nominalphrase (NP) am häufigsten in den drei analysierten Sprachen vorkommt. Mehr als die Hälfte aller Filmtitel im Englischen $(74,33 \%)$ und im Deutschen $(64,67 \%)$ weist die Struktur einer Nominalphrase auf. Die ins Thailändische übersetzten Filmtitel umfassen mit 39,67\% ebenfalls den größten Anteil. Anzumerken sei jedoch, dass im Vergleich zu den beiden europäischen Sprachen, nominale Filmtitel einen viel niedrigeren Anteil aufweisen. Der Konstruktionstyp „Gemischt“ (M) kommt mit 31,67\% im Thailändischen, 22,33\% im Deutschen, und 11,67\% im Englischen in den drei untersuchten Sprachen an zweite Stelle. Es handelt sich bei dieser Kategorie um die Titel, die mehr als einen sprachlichen Konstruktionstypen aufweisen. Des Weiteren wird die gemischte Titelstruktur genauer analysiert.

Auch bei der drittgrößten Kategorie liegt keine Abweichung vor. Satzförmige Titel (S) belegen in allen drei analysierten Sprachen den dritten Platz, jedoch mit unterschiedlichen Anteilen: 16\% im Thailändischen, 6,66\% im Englischen und 4,33 in den deutschen Daten. Die anderen Strukturen kommen nur vereinzelt vor.

Zwar liegt kein einziger Beleg für die Kategorie „Interjektion“ (Interj) vor, so lässt sich diese Wortart doch in der thailändischen Sprache finden. Sie tritt nur mit einer anderen syntaktischen Form auf, weshalb diese Struktur unter der Kategorie „Gemischt“" subsummiert wird.

Im Folgenden werden verschiedene Beispiele für die oben genannten syntaktischen Titelstrukturen in der Reihenfolge ihrer Vorkommenshäufigkeit geschildert. Da die Mischform einer näheren Betrachtung bedarf, wird sie ausnahmsweise erst nach anderen Strukturen dargestellt.

Zuerst ergibt sich die Nominalphrase als die am meisten angewendete Titelstruktur in allen drei untersuchten Sprachen. Ein nominaler Filmtitel wird durch ein Nomen als bestimmtes Element charakterisiert (Hentschel 2010: 198), wie die folgenden Beispiele zeigen:

Zootopia (E_2016_31)

Zoomania (D_2016_31)

nakh o:n sàt mahă: sanùk (T_2016_31)

Stadt Tier super spaßig

'Die spaßige Tierstadt'

Als nächstes werden Beispiele von satzförmigen Titeln aufgezeigt, da sie sowohl im Deutschen $(13,67 \%)$ als auch im Thailändischen $(4,33 \%)$ auf dem dritten Platz stehen, jedoch sieht man, dass sie im Deutschen einen größeren Anteil bildet. Hier sind einige Beispiele davon: 
First They Killed My Father (E_2017_27)

Solange ich atme (D_2017_26)

me:kha: thalòm lök (T_2017_96)

Wolke zerstören Welt

'Wolken zerstören die Welt'

Normalerweise sind Sätze sprachliche Konstrukte, die folgende wichtige Merkmale aufweisen: Sie erhalten ein finites Verb, sind autonom und eignen sich in besonderer Weise zur Vereindeutlichung von Sprechakten (Engel 1988: 180). Jedoch lässt sich ausdrücklich anmerken, dass einige untersuchte Filmtitel in die Kategorie verblose Sätze fallen, wie beispielweise in dem englischsprachigen Beispiel Me Before You (E_2016_77). Der Titel enthält zwar kein finites Verb, aber hier liegt eine satzäquivalente Konstruktion vor, weshalb der Titel hier als satzförmig kategorisiert wird.

Die Verbalphrase liegt im Englischen mit 16 Titeln (5,33\%) auf dem dritten Platz, im Deutschen sowie im Thailändischen kommt sie gleichermaßen an vierte Stelle, jedoch weisen verbale Filmtitel im Thailändischen mit 9,67\% einen größeren Anteil auf als diejenigen im Deutschen (4\%). Im Folgenden sind Beispiele von verbalen Filmtiteln:

Kidnap (E_2017_98)

Kidnap (D_2017_98)

lâ: jùt narók (T_2017_98)

jagen stoppen Hölle

'Jagen, um die Hölle zu stoppen'

Präpositionalsphrasen kommen im untersuchten Korpus in allen drei Sprachen selten vor. Im Thailändischen treten sie jedoch häufiger als im Deutschen und im Englischen auf (T 1,33\%, D1\%, E 0,67\%). Folgendes sind Beispiele für diese Titelstruktur:

Beyond Skyline (E_2017_77)

Im Herzen der See (D_2015_13)

d̀̀: khraj sàk khon (T_2016_51)

für jemand nur Mensch

'Für...nur jemanden'

Adjektivphrasen kommen in allen drei Sprachen ebenfalls selten vor. Es handelt sich außerdem zum Teil um denselben Film:

Furious 7 (E_2017_32)

Fast \& Furious 7 (D_2017_32)

rew re:y thalú? narók 7 (T_2017_32)

schnell stark durch Hölle 7

'Schnell und stark durch die Hölle 7'

Die Adverbphrase gilt für die deutschen Übersetzungsfilmtitel, ähnlich wie Adjektivphrasen und Präpositionalphrasen, als die am wenigsten angewandte Struktur, im Englischen kommt sie jedoch häufiger als die Präpositionalphrase vor. Hingegen kommt diese Struktur im Thailändischen kein einziges Mal vor. Es sei hier zusätzlich anzumerken, dass es sich bei den gefundenen 
deutschen Belegen weniger um Adverbphrasen, sondern um adverbiale Filmtitel handelt, wie im folgenden Beispiel ersichtlich ist:

47 Meters Down (E_2017_16)

47 Meters Down (D_2017_16)

Für immer Adaline (D_2015_74)

Der deutsche Ausdruck für immer lässt sich formal als Präpositionalphrase bezeichnen. Jedoch handelt es sich bei den beiden Fällen um einen adverbialen Filmtitel und wird in der vorliegenden Analyse der Struktur Adverbphrase zugeordnet.

Im Korpus lässt sich die Mischung der syntaktischen Titelstruktur auf dem zweiten Platz finden, die in Tabelle 5 unter ,gemischt“ (M) dargestellt wird. Da es sich bei diesem Konstruktionstyp um mehr als eine Konstruktion innerhalb eines Filmtitels handelt, soll die Struktur dieser Filmtitel näher betrachtet werden. Die 194 Filmtitel (dt. 67, th. 92, engl. 35) unter dieser Kategorie weisen insgesamt 27 Mischungsformen auf, die in verschiedenen prozentualen Anteilen berechnet werden, welche in der folgenden Tabelle dargestellt werden:

\begin{tabular}{|c|c|c|c|}
\hline \multirow{2}{*}{$\begin{array}{c}\text { Gemischte Formen } \\
\text { der Konstruktions- } \\
\text { typen }\end{array}$} & \multicolumn{3}{|c|}{$\begin{array}{c}\text { Anzahl der gemischten Formen } \\
\text { der Konstruktionstypen }\end{array}$} \\
\hline & Deutsch & Thailändisch & Englisch \\
\hline 1. NP + NP & $\begin{array}{c}47 \\
(15,67 \%)\end{array}$ & $\begin{array}{c}37 \\
(12,33 \%)\end{array}$ & $\begin{array}{c}27 \\
(9 \%)\end{array}$ \\
\hline 2. NP + VP & $\begin{array}{c}1 \\
(0,33 \%)\end{array}$ & $\begin{array}{c}19 \\
(6,33 \%)\end{array}$ & $\begin{array}{c}1 \\
(0,33 \%)\end{array}$ \\
\hline 3. NP+S & $\begin{array}{c}9 \\
(3 \%)\end{array}$ & $\begin{array}{c}7 \\
(2,33 \%)\end{array}$ & $\begin{array}{c}3 \\
(1 \%)\end{array}$ \\
\hline 4. NP + Adj.P & $\begin{array}{c}4 \\
(1,33 \%)\end{array}$ & $\begin{array}{c}2 \\
(0,67 \%)\end{array}$ & $\begin{array}{c}1 \\
(0,33 \%)\end{array}$ \\
\hline 5. NP + NP + NP & - & $\begin{array}{c}3 \\
(1 \%)\end{array}$ & $\begin{array}{c}1 \\
(0,33 \%)\end{array}$ \\
\hline 6. NP + Adv.P & $\begin{array}{c}1 \\
(0,33 \%)\end{array}$ & - & - \\
\hline 7. NP + PP & $\begin{array}{c}1 \\
(0,33 \%)\end{array}$ & $\begin{array}{c}1 \\
(0,33 \%)\end{array}$ & - \\
\hline 8. NP + NP + S & - & $\begin{array}{c}1 \\
(0,33 \%)\end{array}$ & - \\
\hline $\begin{array}{l}\text { 9. } \mathrm{NP}+\mathrm{NP}+\mathrm{NP}+ \\
\mathrm{NP}\end{array}$ & - & $\begin{array}{c}1 \\
(0,33 \%)\end{array}$ & - \\
\hline 10. NP + S + VP & - & $\begin{array}{c}1 \\
(0,33 \%)\end{array}$ & - \\
\hline 11. NP + VP + NP & - & $\begin{array}{c}1 \\
(0,33 \%)\end{array}$ & - \\
\hline 12. $\mathrm{NP}+\mathrm{VP}+\mathrm{VP}$ & - & $\begin{array}{c}2 \\
(0,67 \%)\end{array}$ & - \\
\hline
\end{tabular}




\begin{tabular}{|c|c|c|c|}
\hline \multirow{2}{*}{$\begin{array}{l}\text { Gemischte Formen } \\
\text { der Konstruktions- } \\
\text { typen }\end{array}$} & \multicolumn{3}{|c|}{$\begin{array}{l}\text { Anzahl der gemischten Formen } \\
\text { der Konstruktionstypen }\end{array}$} \\
\hline & Deutsch & Thailändisch & Englisch \\
\hline 13. NP + VP + AdjP & - & $\begin{array}{c}1 \\
(0,33 \%)\end{array}$ & - \\
\hline 14. VP + NP & $\begin{array}{c}1 \\
(0.33 \%)\end{array}$ & $\begin{array}{c}3 \\
(1 \%)\end{array}$ & - \\
\hline 15. VP+VP & - & $\begin{array}{c}3 \\
(1 \%)\end{array}$ & - \\
\hline 16. VP + AdjP & - & $\begin{array}{c}1 \\
(0,33 \%)\end{array}$ & - \\
\hline 17. $\mathrm{VP}+\mathrm{S}$ & - & $\begin{array}{c}2 \\
(0,67 \%)\end{array}$ & - \\
\hline 18. VP + VP + VP & - & $\begin{array}{c}1 \\
(0,33 \%)\end{array}$ & - \\
\hline 19. VP + VP + AdjP & - & $\begin{array}{c}1 \\
(0,33 \%) \\
\end{array}$ & - \\
\hline 20. AdjP + NP & $\begin{array}{c}1 \\
(0,33 \%)\end{array}$ & - & $\begin{array}{c}1 \\
(0,33 \%)\end{array}$ \\
\hline 21. AdjP + S & - & $\begin{array}{c}1 \\
(0,33 \%)\end{array}$ & - \\
\hline 22. S + NP & $\begin{array}{c}1 \\
(0,33 \%)\end{array}$ & $\begin{array}{c}1 \\
(0,33 \%) \\
\end{array}$ & $\begin{array}{c}1 \\
(0,33 \%)\end{array}$ \\
\hline 23. S + VP & - & $\begin{array}{c}1 \\
(0,33 \%)\end{array}$ & - \\
\hline 24. $S+$ Adj.P & - & $\begin{array}{c}1 \\
(0,33 \%)\end{array}$ & - \\
\hline 25. S + AdvP & $\begin{array}{c}1 \\
(0,33 \%) \\
\end{array}$ & - & - \\
\hline $26 . S+S$ & - & $\begin{array}{c}1 \\
(0,33 \%)\end{array}$ & - \\
\hline 27. Interj $+S$ & & $\begin{array}{c}1 \\
(0,33 \%)\end{array}$ & \\
\hline
\end{tabular}

Tabelle 6: Gemischte Formen der Konstruktionstypen in den drei analysierten Sprachen

Wie aus Tabelle 6 hervorgeht, liegen 10 Formen der gemischten Konstruktionstypen im Deutschen, 24 im Thailändischen und nur 7 in der Originalsprache vor. Die gemischten Konstruktionstypen weisen im Thailändischen also die größte Vielfalt auf. Die obigen Ergebnisse der Kategorisierung erheben keinerlei Anspruch auf statistische Relevanz, weil es sich vor allem um vereinzelte Belege handelt. Trotzdem stehen sie im Einklang mit der ersten Phrase der Titelstrukturanalyse. Die Nominalphrase ist die beliebteste Struktur bzw. die häufigste Strategie, sei es als Ein-Teil-Titel (NP) oder als mehrteilige Titel erschienen (z. B. NP + X; X+ NP). Es liegen insgesamt 16 gemischte Formen vor, die eine Nominalphrase enthält. Bemerkenswert ist außerdem, dass Drei-Teil-Titel nur im Thailändischen nachweisbar sind. 
Im Nachstehenden folgen nur einige Beispiele der am häufigsten gemischten Strukturen aus dem Korpus.

$N P+N P$ :

Avengers: Age of Ultron (E_2015_86)

Bailey - Ein Freund fürs Leben (D_2017_43)

khu:n ?amahit pi: lîu:aktây hò:t (T_2016_72)

Nacht brutal Jahr Wahl grausam

'Brutale Nacht. Grausames Wahljahr'

$\mathrm{NP}+\mathrm{VP}$

Jumanji: Welcome to the Jungle

Jumanji: Willkommen im Dschungel

?ajs ?e:t phàcon ?ùka:bàt sùt ?onlawey (T_2016_22)

Ice Age konfrontieren Meteorit sehr chaotisch

Ice Age: Chaotische Konfrontation mit Meteoriten

$\mathrm{NP}+\mathrm{S}:$

Pirates of The Caribbean: Dead Men Tells No Tales (E_2017_56)

Vacation - Wir sind die Grinswolds (D_2015_38)

ks:lısa:n tháy càkawa:n penkhǒ:y thr: (T_2017_79)

Colossal ganz Universum gehören du

'Colossal: Das ganze Universum gehört dir'

\section{$5 \quad$ Fazit und Ausblick}

Zielsetzung dieser Arbeit war es, die originalen und übersetzten Hollywoodfilmtitel im Zeitraum von 2015 bis 2017 durch die Analyse von Titellänge, Übersetzungstechnik und sprachlicher Titelstruktur zu untersuchen. Zusammenfassend lässt sich sagen, dass bei der Länge von untersuchten Filmtiteln ein bis zwölf Wörter herausgefunden werden. In den beiden europäischen Sprachen verwendet man Zwei-Wort-Titel am öftesten, während im Thailändischen der Vier-Wort-Titel am häufigsten erscheint. Die verschiedene Titellänge kann u. E. sowohl auf die sprachtypologischen, als auch auf die kulturellen Unterschiede unter den drei untersuchten Sprachen und Kulturräumen zurückgeführt werden. Während die englischen und deutschen Filmtitel häufig nur zwei Wörter enthalten, werden bei der thailändischen Sprache, die eine isolierende Sprache ist, mehr Wörter hinzugefügt, um die Bedeutung zu erweitern. Darüber hinaus gehören sowohl die englisch- als auch die deutschsprachigen Publikumskreise zur westlichen Kultur, während sich das thailändische Publikum in einem anderen Kulturraum befindet, weswegen die Filmtitel im Thailändischen häufig mehr Wörter als die originalen und deutschsprachigen Titel benötigten, um mehr Informationen zu geben.

Den übersetzten Titeln lassen sich zehn Übersetzungsmethoden zuordnen. Die Originalfilmtitel beizubehalten lässt sich im Deutschen am häufigsten finden. Auch dies zeigt die kulturelle Nähe zwischen dem deutschen und dem englischen Zielpublikum im Unterschied zum thailändischen, weshalb die Titelidentitäten bei deutschen Filmtiteln besser funktionieren und häufiger vorkommen. Die Ergebnisse beleuchten außerdem ein ähnliches Bild wie bei anderen Studien, 
die sich nur mit europäischen Sprachen beschäftigten. Bei Bouchehri (2008) ist die Identität der Filmtitel am häufigsten sichtbar (30,3\%). Ähnlich bemerkt Schubert (2004: 257), dass von 1984 bis 2002 fast die Hälfte der Filmtitel identisch übernommen wurde (47,1\%). In Übereinstimmung mit Schubert geht dies auf die Dominanz der englischen neuen Medien und Englisch als Weltsprache zurück. Zudem wirken sich die Einflüsse von anderen englischen Medien, wie beispielsweise Musik, Podcasts oder Websites im Internet auf die Sprachanerkennung des deutschen und des thailändischen Publikums aus.

Die Übernahme der Originaltitel tritt bei der thailändischen Version ebenfalls zwar häufig auf, aber erst an zweiter Stelle und mit einem großen Abstand von dem ersten Platz, der Umtitelung in der Zielsprache. Bemerkenswerterweise weist dieses Ergebnis Unterschiede zu englischsprachigen Filmtiteln auf, die ins Chinesische übersetzt worden sind, obwohl Chinesisch und Thailändisch sprachtypologisch ähnlich sind. In einer Studie von Lu (2014) wurde beispielsweise die Wort-für-Wort-Übersetzung als die meist verwendete Übersetzungsmethode nachgewiesen. Auch in einer Studie zu Strategien und Problemen beim Übersetzen von Titeln literarischer Werke aus dem Deutschen ins Thailändische (O'charoen 2014) erscheint die wörtliche Übersetzung am häufigsten. Die Umtitelung in der Zielsprache scheint für das Genre Filmtitel besonders zu sein. Dies steht mit Thongwans (2012) Ergebnis im Einklang, bei dessen untersuchten Übersetzungen englischer Filmtitel ins Thailändische Titelinnovationen auf dem ersten Platz stehen.

In dem vorliegenden analysierten Korpus verwendet man im Thailändischen zudem Wortspiele häufiger als im Deutschen, um die genauere Bedeutung der Filme zu beschreiben. Außerdem kommen andere Translationskategorien in beiden übersetzten Filmtiteln vor, beispielsweise die wörtliche Übersetzung mit Titelgefüge oder die Reduktion mit Titelgefüge. Die Mischtechnik wird im thailändischen Korpus am häufigsten festgestellt.

Im Hinblick auf die sprachlichen Konstruktionstypen können insgesamt 7 Gruppen kategorisiert werden. Zweifellos ist die Nominalphrase die am öftesten angewendete Titelstruktur in allen drei Sprachen. Wegen der inhaltlichen Deutlichkeit der Nomen, die sich in der Regel auf Entitäten, besonders Konkreta, beziehen, benutzt man sie häufiger als andere Formen. Die Rezipienten können den Filminhalt besser begreifen, wenn der Name des Films mit einem Substantiv betitelt wird.

Die Unterschiede zwischen den Sprachen Englisch, Deutsch und Thailändisch und den drei unterschiedlichen Publikumskreisen könnten in der Tat Raum für eine intensive Auseinandersetzung mit dem Aspekt Sprach- und Kulturtransfer anbieten, was allerdings nicht im Rahmen dieser Untersuchung vorgesehen ist, weil sie nur als eine kleine Pilotstudie gilt, bei der zum ersten Mal typologisch verschiedene Sprachen miteinander verglichen werden. Deshalb beschränkt sich die Studie nur auf messbare und objektiv analysierbare Elemente wie Länge, Übersetzungsmethoden und die syntaktische Ebene der Filmtitel. Jedoch soll die vorliegende Arbeit den Weg für weitere Untersuchungen bahnen. Eine Erweiterung wäre, die deutschen und thailändischen Originalfilmtitel miteinzubeziehen. Titel von Filmen, die in Thailand und in Deutschland produziert werden und direkt für die beiden Publikumskreise erdacht werden, könnten entweder ein ähnliches oder ein gegensätzliches Bild zu übersetzten Titeln aufweisen. Auch weitere Forschungen im Hinblick auf den Zusammenhang zwischen Filmgenres und 
sprachlichen Aspekten wie Länge der Filmtitel, Übersetzungsmethoden und auch Titelstrukturen in unterschiedlichen Zielpublikumskreisen wären ebenso interessant. Nicht zuletzt könnte die syntaktische Analyse von Original- und Übersetzungsfilmtiteln mit mehr Daten erweitert werden, sei es mit oder ohne Ergänzung von zu analysierenden Sprachen. Auch zusätzliche sprachlichen Aspekte könnten miteinbezogen werden. Die satzförmigen Filmtitel könnten beispielsweise verschiedenen Satzarten zugeordnet werden, um die Filmtitelübersetzung detaillierter im Hinblick auf die Textfunktion zu untersuchen, was bei sprachtypologisch und kulturell verschiedenen Sprachen ebenfalls zu neuen Erkenntnissen führen könnte.

\section{Literaturverzeichnis}

Bouchehri, Regina (2008): Filmtitel im interkulturellen Transfer. Berlin: Frank \& Timme.

Brückner, Anne (2012): Filmtitelübersetzung. Potsdam: Publikationsserver der Universität Potsdam.

Engel, Ulrich (1988): Deutsche Grammatik. Heidelberg: Groos.

Hentschel, Elke (ed.) (2010): Deutsche Grammatik. Berlin/New York: de Gruyter.

Lu, Wenjing (2014): “An Analytical Study of Film Title Translation into Chinese Language”. In: Proceedings of National Research Conference 2014, Rangsit University, April 3, 2014: 708-715.

Masae, Rusnee et al. (2018): “An Analysis of Strategies Used in Translations Hollywood Movie Titles From English to Thai”. In: Proceedings of the $9^{\text {th }}$ Hatyai National and International Conference, Hatyai University, July 20-21, 2018: 111-118.

Nord, Christiane (1991): Textanalyse und Übersetzen: theoretische Grundlagen, Methode und didaktische Anwendung einer übersetzungsrelevanten Textanalyse. Heidelberg: Gross.

O'charoen, Thanomuan (2014): Strategies and Problems of Title Translation of German Literary Works into Thai. Forschungsbericht. Bangkok: Chulalongkorn University.

Schubert, Christoph (2004): Die Appellwirkung englischer Filmtitel und ihrer deutschen Neutitel: Techniken interkulturellen Transfers. Tübingen: Narr.

Thongwan, Supawan (2012): Translating American Comedy Film Titles into Thai: Strategies and Analysis. Master Thesis. Silpakorn University. 